\title{
MIPAS Level 1B algorithms overview: operational processing and characterization
}

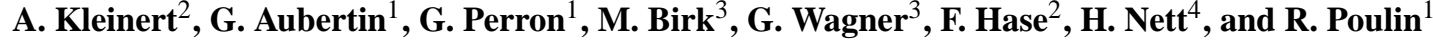 \\ ${ }^{1}$ ABB Bomem Inc., 585 Blvd. Charest East, Québec, G1K 9H4, Canada \\ ${ }^{2}$ Forschungszentrum Karlsruhe GmbH, Institut für Meteorologie und Klimaforschung, Postfach 3640, 76021 Karlsruhe, \\ Germany \\ ${ }^{3}$ Remote Sensing Technology Institute, German Aerospace Centre, Muenchner Str. 20, 82234 Wessling, Germany \\ ${ }^{4}$ ESTEC, Kepplerlaan 1, Noordwijk, The Netherlands
}

Received: 22 September 2006 - Published in Atmos. Chem. Phys. Discuss.: 20 October 2006

Revised: 2 February 2007 - Accepted: 13 February 2007 - Published: 28 February 2007

\begin{abstract}
This paper gives an overview of the MIPAS Level 1B (L1B) processor whose main objective is to calibrate atmospheric measurements radiometrically, spectrally and geo-located. It presents also the results of instrument characterization done on ground and during the first years inflight. An accurate calibration is mandatory for high quality atmospheric retrievals. MIPAS has shown very good performance and stability. The noise equivalent spectral radiance ranges from 3 to $50 \mathrm{nW} /\left(\mathrm{cm}^{2} \mathrm{srcm}^{-1}\right)$ and is well within the requirements over nearly the whole spetral range. The systematic radiometric error is estimated to be within 1 or $2 \%$ in most situations.
\end{abstract}

\section{Introduction}

MIPAS is a Michelson Interferometer based on the principle of Fourier Transform and designed to measure with high resolution and high spectral accuracy the emission of infrared radiation from the atmosphere in the spectral range from 4.15 to $14.6 \mu \mathrm{m}\left(685-2410 \mathrm{~cm}^{-1}\right)$ (Fischer et al., 2006; Fischer et al., 2000; Endenman, 1999). This paper covers the full resolution mission from 2002 to March 2004.

The MIPAS instrument is designed to observe the horizon with an instantaneous field of view that corresponds at the tangent point to $3 \mathrm{~km}$ in vertical direction and $30 \mathrm{~km}$ in horizontal direction. It can make measurements in rearward viewing (anti-flight direction) to cover horizontally a $35 \mathrm{deg}$ range, or in sideways viewing to cover a $30 \mathrm{deg}$ wide range in anti-sun direction. Most measurements are made in rearward viewing.

Correspondence to: G. Perron

(gaetan.p.perron@ca.abb.com)
The MIPAS interferometer provides two-sided interferograms with a maximum optical path difference of $20 \mathrm{~cm}$. It has a dual port configuration with two input ports and two output ports. Only one input port is needed to acquire data from a given scene. The second input port is designed to look at a cold target in order to minimize its contribution to the signal. The cold target is a cold plate of high emissivity cooled at $70 \mathrm{~K}$. Each output port is equipped with four detectors covering different spectral bands. The signals detected at both output ports are similar and they are combined in some frequency bands to improve signal-to-noise ratio. The eight detectors are split into five bands, each band being covered by one or two specific detectors. Due to the limited data rate, the measured interferograms are filtered and decimated. The filtering before decimation prevents noise from out-of-band spectral regions to be aliased into the spectrum. The spectral coverage of the eight detectors and the five bands, together with the decimation factor, is given in Table 1.

A nominal measurement sequence consists in a series of 17 high resolution atmospheric scene measurements made at different tangent heights starting at $68 \mathrm{~km}$ tangent height and descending to $3 \mathrm{~km}$. The observation geometry is displayed in Fig. 1. The elevation steps go from $8 \mathrm{~km}$ step at high altitude to $3 \mathrm{~km}$ step at low altitude. The sweep duration at high resolution is $4.45 \mathrm{~s}$.

Before being transmitted to the ground the detected signal is amplified and analog filtered, digitized, numerically filtered and decimated, equalized and combined, bit truncated and finally packetized. The Level 0 (L0) product is then processed by the L1B ground segment processor to generate geo-located spectra that are radiometrically and spectrally calibrated. 
Table 1. Spectral bands and contributing detectors in nominal operation.

\begin{tabular}{ccccc}
\hline Detector & $\begin{array}{c}\text { Optical Range } \\
{\left[\mathrm{cm}^{-1}\right]}\end{array}$ & Band & $\begin{array}{c}\text { Decimation } \\
\text { Factor }\end{array}$ & $\begin{array}{c}\text { Optical Range } \\
{\left[\mathrm{cm}^{-1}\right]}\end{array}$ \\
\hline A1 & $685-995$ & A & 21 & $685-970$ \\
A2 & $685-1193$ & AB & 36 & $1020-1170$ \\
B1 & $995-1540$ & B & 22 & $1215-1500$ \\
B2 & $1193-1540$ & C & 30 & $1570-1750$ \\
C1 , C2 & $1540-1780$ & D & 11 & $1820-2410$ \\
D1 , D2 & $1780-2410$ & D & & \\
\hline
\end{tabular}

Table 2. Notation for the radiometric calibration.

\begin{tabular}{lcccc}
\hline Spectrum & Cold Source & Hot Source & Scene & Units \\
\hline Radiance & 0 & $L^{b b}$ & $L^{x}$ & $\mathrm{nW} /\left(\mathrm{cm}^{2} \mathrm{srcm}^{-1}\right)$ \\
Observed & $S^{d s}, S^{c}$ & $S^{b b}$ & $S^{x}$ & Arbitrary \\
\hline
\end{tabular}

Note: $S^{c}$ is the closest offset calibration (deep space) to the scene to calibrate, whereas $S^{d s}$ is the deep space measurement used for the calculation of the gain function.

\section{Level 1B algorithms}

For MIPAS, the output of the L1B ground processor is an atmospheric spectrum showing radiance as a function of wavenumber. Calibration refers not only to the assignment of absolute radiance values to the $y$-axis but also to the assignment of absolute wavenumbers to the $\mathrm{x}$-axis.

Three types of calibration for MIPAS are required:

Radiometric Calibration:

The process of assigning absolute values in radiance units, (noted [r.u.] expressed in $\left(\mathrm{nW} /\left(\mathrm{cm}^{2} \mathrm{srcm}^{-1}\right)\right)$ ) to the intensity axis (y-axis) with a specified accuracy. The radiometric calibration implies the knowledge of a certain spectral calibration.

\section{Spectral Calibration:}

The process of assigning absolute values in $\mathrm{cm}^{-1}$ to the wavenumber axis (x-axis) with a specified accuracy.

\section{Line of sight (LOS) Calibration:}

The process of assigning a calibrated LOS pointing angle to a given atmospheric spectrum with a specified accuracy. These calibrated angles combined with spacecraft position are used to determine the geo-location of the tangent point.

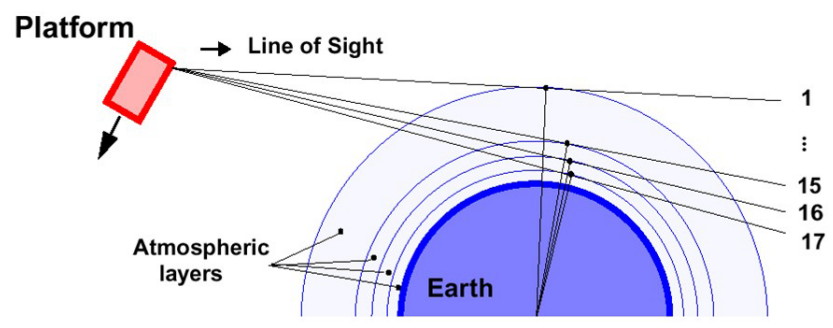

Fig. 1. Observation geometry of MIPAS.

The details of the Level 1B algorithms are described in PO-RP-BOM-GS-0003 4I (2004); PO-TN-BOM-GS-0012 (2002).

\subsection{Radiometric calibration}

The radiometric calibration uses the approach described by Revercomb et al. (1988). It is performed using two known radiation sources (a hot source and a cold source). The hot source is an internal calibration blackbody, while the deep space serves as cold source. Two sets of measurement scenarios are required:

1. Deep space measurements followed by scene measurements to correct the scene for self-emission of the instrument. Deep space measurements are done frequently (once every four elevation scans) in order to account for changing self-emission of the instrument due to temperature variations along the orbit.

2. Blackbody (BB) measurements followed by an equivalent number of deep space measurements to calculate the radiometric gain function. The gain calibration is done once per week.

Using the calibration blackbody as the hot source and the deep space as the cold source and using the notation given in Table 2, the radiometric gain $G$ is defined as:

$G=\frac{L^{b b}}{S^{b b}-S^{d s}}$

The radiometric gain is a complex function taking in consideration that MIPAS interferograms are filtered by a complex numerical filter (see Sect. 2.4.1). In the L1B processing, the forward and reverse sweep directions are always treated separately to take into account that the phase is different in forward and reverse (see Sect. 2.4.2).

The expression for radiometric calibration of a scene becomes

$L^{x}=G \cdot\left(S^{x}-S^{c}\right)$

In order to improve the signal to noise ratio, the offset calibration is obtained from 6 successive deep space measurements ( 3 forward and 3 reverse). The determination of the 
gain is composed of 300 coadded measurements of the MIPAS internal calibration blackbody source and an equivalent number of coadded deep space measurements for each sweep direction of the interferometer.

\subsection{Spectral calibration}

Spectral calibration is performed using standard measurements from the atmosphere. Particular spectral lines are retrieved in the observed spectrum and the known values of their wavenumbers are used to establish the assignment of the wavenumber to the index of spectral data points. The spectral calibration is used for the wavenumber assignment of all subsequent scene and gain measurements until the new spectral calibration is performed after 4 elevation scans.

In summary, the spectral calibration is based on the following assumptions:

- The spectral calibration includes the spectral shift and is performed without any ILS (Instrumental line shape) deconvolution.

- A minimal number of 4 scene measurements are sufficient for a proper spectral calibration. The scenes chosen are geo-located at a tangent height range from 29 to $35 \mathrm{~km}$.

- The spectral calibration shift function is applied throughout the spectral range. It is assumed that the definition of the optical axis is common to all 8 detectors. It is also assumed that the residual misalignment between the two output ports is low enough so that the difference in wavenumber is negligible.

- Appropriate spectral lines are identified and the value of their wavenumber is available for ground processing by a Microwindow Dictionary Auxiliary data.

\subsection{Geolocation}

It is assumed that commanded elevation angles are only partially corrected with respect to known pointing errors according to the best knowledge based on on-ground characterization and LOS calibration measurements. The remaining elevation error, obtained from weekly LOS calibration measurements, is computed in the payload data ground segment (PDS) and used to correct in measurement mode the measured elevation angles. The corrected elevation angles and the measured azimuth angles are used to compute the geolocation (height/longitude/latitude) of the actual scene (target).

The L1B processor computes the actual pointing error with respect to pitch and roll at the time of scene measurement. It then computes for each scene the actual azimuth angle. Pointing errors in azimuth are not calibrated during flight operation. However, a correction based on on-ground characterization/ alignment measurements must be performed.
The correction on the measured elevation angle of a scene is computed by using the estimated bias and harmonic pointing errors for rearward and sideways measurements, the commanded azimuth angle, and the time of data acquisition.

The geolocation is calculated using orbit and pointing libraries provided by ESA which require as inputs the orbit state vector and the corrected pointing angles (PO-IS-DMSGS-00559, 2005).

\subsection{Other considerations}

\subsubsection{Filtering and decimation}

An observed interferogram is basically a real and symmetrical function. It may be slightly asymmetrical due to beamsplitter emission. In order to reduce data throughput, interferograms are filtered and decimated to limit the spectral contents to the region of interest. MIPAS complex numerical filter has the advantage of allowing a decimation factor two times larger than with a real numerical filter. It is then possible to better optimize the decimation factor. On the other hand, one complex interferogram is produced by the numerical filtering, made of one real and one imaginary part.

\subsubsection{Fringe count errors}

The basic ground processing for MIPAS contains no explicit phase correction or compensation. For a given interferometer sweep direction, it is assumed that the gain and offset calibrations and also the scene measurements have the same phase relationship, i.e. they are sampled at precisely the same intervals. This sampling is determined by a fringe counting system using a reference laser source within the interferometer subsystem, with the fringe counts forming a "clock" signal to the ADC in the on-board signal processing electronics (SPE). The fringes trigger the sampling of the IR interferogram. If, for any reason, a fringe is lost, then the phase of subsequent measurements will be affected and, if these are calibrated using a gain or offset measurement taken before the occurrence of the fringe loss, then errors will be introduced into the final spectrum. The L1B processing detects and corrects for fringe losses by analyzing the residual phase of the spectrum for bands $\mathrm{AB}$ and $\mathrm{C}$ following radiometric calibration. Hence there is no specific measurement required as part of calibration for this aspect. It is assumed that fringe count errors occur only at turnaround and not within an interferogram, therefore the interferograms are used after the fringe count error correction.

\subsubsection{Detector spikes}

Spikes due to cosmic ray going through the detector or transmission error are expected in MIPAS measurement. Onorbit data shows that around $1 \%$ of interferograms are affected. For calibration measurements, data containing detected spikes are discarded in order to not propagate in 
Table 3. MIPAS L1B processor input auxiliary file.

\begin{tabular}{lll}
\hline Type & Identifier & Name \\
\hline Calibration & MIP_CG1_AX & Gain calibration \\
& MIP_CL1_AX & LOS calibration \\
& MIP_CS1_AX & ILS and spectral calibration \\
& MIP_CO1_AX & Offset calibration \\
Auxiliary & MIP_CA1_AX & Instrument characterization data \\
& MIP_PS1_AX & Processing parameters \\
& MIP_MW1_AX & Microwindow dictionary \\
\hline
\end{tabular}

"good" scene measurements during calibration. For scene measurements, a simple algorithm removes the spike by taking the mean of immediate non-affected data points and raises a L1B product confidence data (PCD) flag to warn the user about the quality of data.

\subsubsection{Detector non-linearity}

Detectors from channels A1, A2, B1 and B2 are affected by non-linearity, i.e., detector response is not a linear function of incident photon flux. An algorithm is needed to correct the non-linearity to bring MIPAS radiometric accuracy within requirement. Detector non-linearity was characterized onground and details are given in Sect. 4.4 for the non-linearity characterization.

The first step for the non-linearity correction is to compute the total incoming photon flux (in digitalization units [d.u.]) at the origin of the present interferogram. This meaningful parameter can be estimated with the minimum and maximum values of the digitized interferogram before filtering and decimation. These values are contained in the data stream. The scaling factor applied to the scene is computed for each channel from this total photon flux and the responsivity coefficients of each detector. Blackbody and deep space interferograms are scaled likewise.

\subsubsection{Electronic responsivity scaling}

In the MIPAS instrument, each interferogram must be scaled to account for the current preamplifier gain settings at the time of measurement. The only scaling applied to the measurements takes into account the commanded gain setting that optimizes the detector signal at the ADC level. During commissioning phase, the best commanded gain settings were calculated by measuring the variations of the maximum and minimum ADC values over several orbits for different types of scenes from high altitude to low altitude, for deep space measurements and blackbody measurements.

\subsubsection{Instrument Line Shape (ILS) retrieval}

The chosen ILS retrieval method is called the "Parametric ILS Fitting Method" (PIFM). This method proceeds with a theoretical ILS, obtained by a modelization with a limited number of parameters, convolved with the theoretical line and iteratively fit to the experimental data. Two parameters of the full ILS model are adjusted. The selected parameters are part of the characterization of the interferometer alignment status, one parameter is the amount of linear shear across the scan direction and the other parameter is the misalignment between the optical axis and the center of the fieldof-view. Nominal scene measurements are used for the ILS retrieval. Since ILS retrieval is a very intensive CPU process, the ILS retrieval is limited to basically 5 scenes coadded at a given tangent height specified in the Microwindow dictionary to improve the signal to noise ratio. The steps involved for the ILS retrieval are:

- Selection of specific microwindows containing precisely one reference peak of well-known wavenumber.

- Generation of the reference theoretical spectral line corresponding to this microwindow.

- Fitting an ILS to the incoming radiometrically calibrated spectrum by minimizing residuals between the reference line and the parametric ILS.

Only one ILS retrieval is processed per orbit. The result is part of the L1B product but it is not used when processing near real time L2 data product. It was shown that coaddition of 5 scenes is not sufficient to have stable ILS parameters retrieval. For this reason, L2 processing uses an auxiliary data containing ILS parameters that have been obtained by coadding equivalent scenes from a full orbit, instead of ILS parameters from the L1B product.

\section{Level 1B processing}

The L1B processor requires 2 input types: the Level 0 product containing the measurement data, and a set of auxiliary data files (see Table 3) to calibrate the measurement data. The output of the L1B processor is a L1B product containing the calibrated scene measurements.

\subsection{Level 0 input products}

The L0 product contains time-ordered source packets from the MIPAS instrument. Each source packet corresponds to reconstructed unprocessed instrument data. Nominally, each target measurement consists of 6 interferograms (one for each detector or band $\mathrm{A} 1, \mathrm{~A} 2, \mathrm{AB}, \mathrm{B}, \mathrm{C}$ and $\mathrm{D})$ separated into blocks and individually bit-truncated. The LO product contains the scene, blackbody, and deep scene measurements for more or less one full orbit. For each L0 product received, the L1B processing generates one L1B product per orbit.

In addition to each measurement data sweep, the LO product includes a full record of the on-board instrument status, as well as instrument-level and platform-level parameters 
such as pointing and timing information. The auxiliary data source packet contains status information from the MIPAS instrument control unit (ICU) and status/parameters from the signal processing electronics (SPE). This auxiliary data is appended to each data measurement.

\subsection{Auxiliary Data Files (ADFs)}

The calibration and characterization data is stored in auxiliary data files (Aubertin et al., 2002). These files are listed in Table 3.

\subsubsection{Calibrations}

The gain calibration ADF (MIP_CG1_AX) is used by the L1B processing to detect and correct for fringe count errors in the reconstituted interferogram of the L0 product and to calibrate radiometrically the calculated spectrum.

The offset calibration ADF (MIP_CO1_AX) contains a reference offset that is mainly used as a quality check for the offset measurements from the L0 file. It is used for calibration only if the closest offset measurement exceeds the quality thresholds defined in the processing parameters (MIP_PS1_AX).

The ILS and spectral calibration ADF (MIP_CS1_AX) is used to calibrate the initial spectra from the first 4 elevation scans in the L0 product. Then a new spectral calibration is calculated every 4 elevation scans and is applied to the subsequent spectra.

The line-of-sight calibration ADF (MIP_CL1_AX) is used to calibrate along the orbit the geo-location of scene measurements in the $\mathrm{L} 0$ product.

The gain and offset calibration ADFs (MIP_CG1_AX and MIP_CO1_AX) are updated weekly to compensate for contamination and temperature effects. All historic ADF files are regenerated when an updated configuration allows gain and offset characterization improvements.

The spectral calibration ADFs (MIP_CS1_AX) are updated on a weekly basis, prior to the gain calibration, as analysis has demonstrated that it results in improved gain calibration, and improvements for processing of the initial scans of each product. The spectral calibration history is reconstructed prior to reconstruction of the gain history.

The line-of-sight calibration ADF (MIP_CL1_AX) is updated monthly to correct for deviations in platform attitude. The calibration history for this file has already been reconstructed for historic deviations in pitch.

\subsubsection{Characterizations and processor parameters}

The instrument characterization (MIP_CA1_AX) contains thermistor, non-linearity, equalization, blackbody, detector, line of sight, filtering and gain setting characterizations.

The processing parameter (MIP_PS1_AX) contains spectral axis definition, sinc interpolation table, standard deviation and rejection thresholds, and conversion matrix.
The microwindow dictionary (MIP_MW1_AX) contains the reference microwindows that are used for spectral calibration and ILS retrieval.

\subsection{Level 1B processing functionality}

The overall processing chain, divided into its high-level functions, is processed in the following order. The ADFs used for each function are indicated between parentheses.

- Load Data from L0 product: this function performs the initial processing of all incoming data from the L0 product. It extracts data packets, calibrates relevant auxiliary data (MIP_CA1_AX and MIP_PS1_AX), and sorts single measurement data according to the type of measurement (i.e, scene, blackbody or deep space).

- Calculate Offset Calibration: this function generates a series of calibrated offsets that will be used by the scene calibration module to calculate the scene radiance. The function performs spikes detection (MIP_PS1_AX), sorts offset data according to the interferometer sweep direction, detects and corrects for fringe count errors (MIP_CG1_AX), coadds interferograms in each band ( 3 forward / 3 reverse), corrects for the detectors nonlinearity (MIP_CA1_AX), equalizes and combines interferograms in band A. It finally assesses the NESR (Noise Equivalent Spectral Radiance) performance and checks the validity of incoming offset measurements (MIP_PS1_AX).

- Calculate Gain Calibration: this function generates the output radiometric gain that is stored in the L1B product. The function performs spikes detection (MIP_PS1_AX), sorts blackbody and deep space measurements according to the interferometer sweep direction, detects and corrects for fringe count errors (MIP_CG1_AX), coadds interferograms to increase the signal to noise ratio, corrects for detector nonlinearity (MIP_CA1_AX), calculates coarse spectra for blackbody and deep space interferograms, calculates the theoretical blackbody radiance from temperature readings corresponding to blackbody measurements (MIP_CA1_AX) and calculates the complex gain ratio. It finally checks for radiometric accuracy of the blackbody and deep space measurements (MIP_PS1_AX). Note that the L1B processing uses the same input gain (MIP_CG1_AX) while processing all measurements in the L0 product. The outcome of the gain calibration must be approved by experts prior to being used by the L1B processor to ensure the quality of calibrated scene spectra. This approval is done once a week.

- Calculate Scene Calibration: this function generates the geo-located, spectrally and radiometrically calibrated scene spectra. The function performs spikes detection (MIP_PS1_AX), sorts scene measurements 
Table 4. MIPAS NESR 0 requirement.

\begin{tabular}{cc}
\hline $\begin{array}{c}\text { Wavenumber } \\
{\left[\mathrm{cm}^{-1}\right]}\end{array}$ & $\begin{array}{c}\mathrm{NESR}_{0} \text { requirement } \\
{\left[\mathrm{nW} /\left(\mathrm{cm}^{2} \mathrm{sr} \mathrm{cm}^{-1}\right)\right]}\end{array}$ \\
\hline $685-970$ & 50 \\
$1020-1170$ & 40 \\
$1215-1500$ & 20 \\
$1570-1750$ & 6 \\
$1820-2410$ & 4.2 \\
\hline
\end{tabular}

according to the interferometer sweep direction, detects and corrects for fringe count errors (MIP_CG1_AX), corrects for detector non-linearity (MIP_CA1_AX), equalizes and combines interferograms in band $\mathrm{A}$, subtracts the closest calibrated offset obtained during the offset calibration, computes scene spectra, corrects the spectral axis for Doppler shift and instrument metrology (MIP_CS1_AX and subsequent spectral calibration calculations), performs spectral interpolation onto a pre-defined uniform spectral axis (MIP_PS1_AX), multiplies the scene spectra by the most recent gain (MIP_CG1_AX). It performs scene measurement quality verification (MIP_PS1_AX) and reports the NESR. Each scene measurement is also calibrated for the geolocation. The function computes the actual pointing error at time of zero path difference crossing of the measurement, the actual azimuth pointing angle, the correction of elevation angle and finally the actual elevation pointing angle (MIP_CL1_AX). It then computes the orbital position of the spacecraft at zero path difference time, computes the tangent height, the longitude and latitude. It estimates the error of the computed tangent height.

- Calculate Spectral Calibration: this function determines the spectral calibration factor for the following elevation scans. It is applied at every 4 elevation scans. It uses as inputs the previously radiometrically calibrated scene obtained during the scene calibration. Specific reference spectral lines are retrieved from the scene measurements (MIP_MW1_AX). Equivalent scenes are coadded to reduce noise, i.e., scenes with altitude included in pre-determined range (MIP_PS1_AX). The function computes the spectral calibration factor based on the known values of the reference line positions and the measured line positions.

- Calculate ILS Retrieval: this function generates once per orbit the ILS retrieval from the radiometrically and spectrally calibrated spectra (MIP_CS1_AX, MIP_CG1_AX). Specific reference spectral lines are retrieved from the scene measurements (MIP_MW1_AX). A theoretical ILS is modelized using a limited number of parameters (MIP_PS1_AX), convolved with the theoretical line and iteratively fits the results onto the experimental data.

- Format L1B product: this function performs the packaging of all the processed spectra and computed calibrations into the Level 1B product.

\subsection{Level 1B product output}

The Level 1B product consists in a set of localized, radiometrically and spectrally calibrated spectra of the atmosphere with various annotated data calibrations, data assessments and data quality validation.

Each sweep generates 5 calibrated spectra (1 for each band) and the sweep information header, all with the same time of measurement. The output data file contains the calibrated spectra with reference to calibration data and to the auxiliary data used during calculations. Each elevation scan header contains data specific for an individual elevation sequence belonging to the current scene.

Details on L0, L1B and auxiliary product format are found in PO-ID-DOR-SY-0032 (2000).

\section{Level 1B characterization}

\subsection{Level 1B requirements}

\subsubsection{Noise}

The noise is quantified by the noise equivalent spectral radiance (NESR). The NESR $t$ is defined as the standard deviation of the measured single sweep spectral radiance taken over $N$ measurements for the input signal of a blackbody at temperature $T$ assuming stationary conditions. The $\mathrm{NESR}_{0}$ is defined as $\mathrm{NESR}_{t}$ with $T$ sufficiently small that the noise contribution from the input signal becomes negligible to the noise contributions from the instrument itself. The $\mathrm{NESR}_{0}$ is evaluated from deep space measurements at $210 \mathrm{~km}$ altitude. Table 4 gives the MIPAS NESR 0 to achieve in the respective bands assuming high spectral resolution.

\subsubsection{Radiometric accuracy}

The radiometric accuracy shall be better than the sum of $2 \times$ $\mathrm{NESR}_{t}$ and $5 \%$ of the source spectral radiance using a blackbody with a maximum temperature of $230 \mathrm{~K}$ as source, in the $685-1500 \mathrm{~cm}^{-1}$ region. It shall be better than the sum of $2 \times \mathrm{NESR}_{t}$ and $3 \%$ of the source spectral radiance using a blackbody with a maximum temperature of $230 \mathrm{~K}$ as source, in the $1570-2410 \mathrm{~cm}^{-1}$ region. This is the requirement, but the goal in the spectral region of $685-970 \mathrm{~cm}^{-1}$ is a radiometric accuracy of $1 \%$ in order to ensure precise temperature retrieval. 


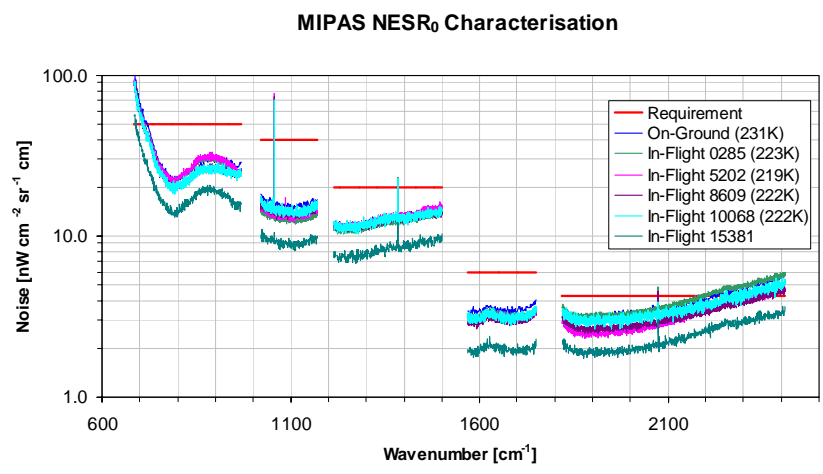

Fig. 2. NESR 0 of MIPAS on ground and in flight.

\subsubsection{Spectral considerations}

The spectral resolution of MIPAS shall be less/equal $0.035 \mathrm{~cm}^{-1}$ throughout the spectral range in high spectral resolution. The spectral resolution is defined as the FullWidth at Half Maximum (FWHM) of the ILS.

The spectral linearity shall be smaller than $0.001 \mathrm{~cm}^{-1}$ throughout the complete spectral range.

The spectral stability of the instrument shall be better than $0.001 \mathrm{~cm}^{-1}$ during at least $165 \mathrm{~s}$ of operation, but the goal is to achieve a spectral stability of $0.001 \mathrm{~cm}^{-1}$ over $24 \mathrm{~h}$ of in-orbit operation.

\subsection{NESR}

The NESR (noise equivalent spectral radiance) quantifies the noise level of the data. The $\mathrm{NESR}_{0}$ is the NESR in absence of signal radiation. For characterization purposes, the NESR 0 is determined by calculating the standard deviation of consecutive high resolution deep space spectra. Furthermore an NESR is calculated for each calibrated spectrum. This NESR is derived from the imaginary part of the calibrated spectrum, by taking the standard deviation of all data points within a spectral interval of $10 \mathrm{~cm}^{-1}$. This NESR is reported on a spectral grid of $10 \mathrm{~cm}^{-1}$.

The $\mathrm{NESR}_{0}$ ranges from $3 \mathrm{nW} /\left(\mathrm{cm}^{2} \mathrm{srcm}^{-1}\right)$ to $50 \mathrm{nW} /\left(\mathrm{cm}^{2} \mathrm{srcm}^{-1}\right)$, depending on the spectral range and channel. The NESR is well within the requirements (Fig. 2), and no systematic evolution of the NESR over time could be observed. The most important effect on the NESR is ice accumulation on the detector system, which may temporarily degrade the NESR by up to $20 \%$ with respect to an ice free detector.

\subsection{Radiometric stability}

\subsubsection{Gain calibration}

A gain function is typically generated once per week, and the last gain function generated is used for calibration. Changes in the gain function are mainly due to ice contamination of

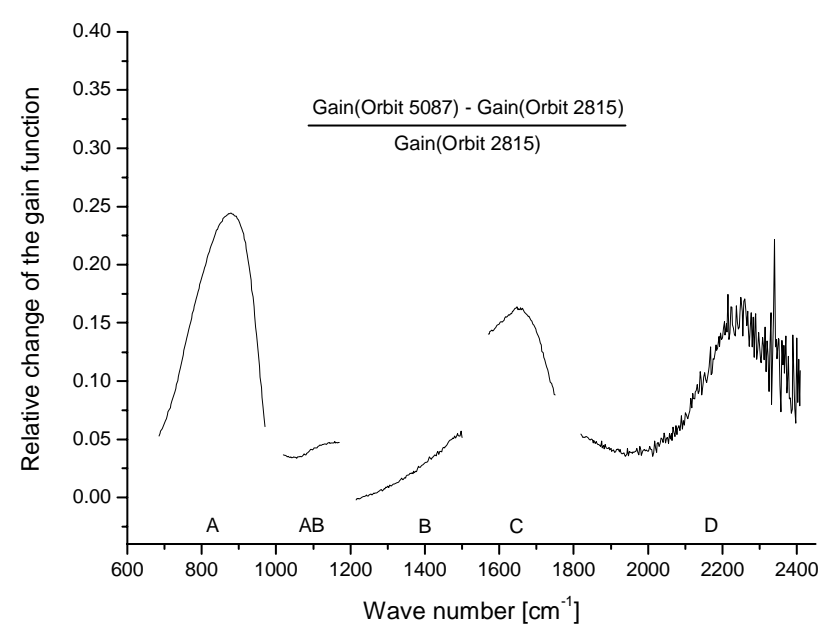

Fig. 3. Relative change of the gain function between orbit 2815 (13.09.2002) and orbit 5087 (02.12.2003).

the detector system, but temperature effects can also be observed. As an example, the relative difference between the two gain functions of orbit 2815 (13.09.2003) and orbit 5087 (02.12.2003) is shown in Fig. 3. The spectral shape of the difference clearly reveals the broadband features of ice. The increase of the gain function is a measure for a decreasing detector signal due to a degraded transmission. Figure 4 shows the increase of the gain function around $870 \mathrm{~cm}^{-1}$ (where the effect of ice is most prominent) relative to the reference orbit 2815. The decontamination intervals, where the gain function decreases again, are clearly visible. On the right axis in the same figure, the maximum change between two consecutive gain functions is plotted. This corresponds to the maximum gain error due to drift of the gain function. This error is typically around 1 to $2 \%$ and slightly decreasing over the lifetime of the instrument. In case of large changes between consecutive gain functions (typically in case of measurement interruptions) the first gain function, which is taken after the interruption, is used for the calibration of the offline data.

\subsubsection{Offset stability}

The instrument offset is much more sensitive to changes in the instrument temperature than the gain function. Therefore, the offset is determined several times per orbit. A small variation of the offset along the orbit can be observed, however, these variations are in the order of 1/10 NESR between two offset measurements and are negligible in the radiometric error budget.

\subsection{Detector non-linearity}

The photoconductive Mercury-Cadmium-Telluride detectors show a non-linear dependence of output signal versus input 


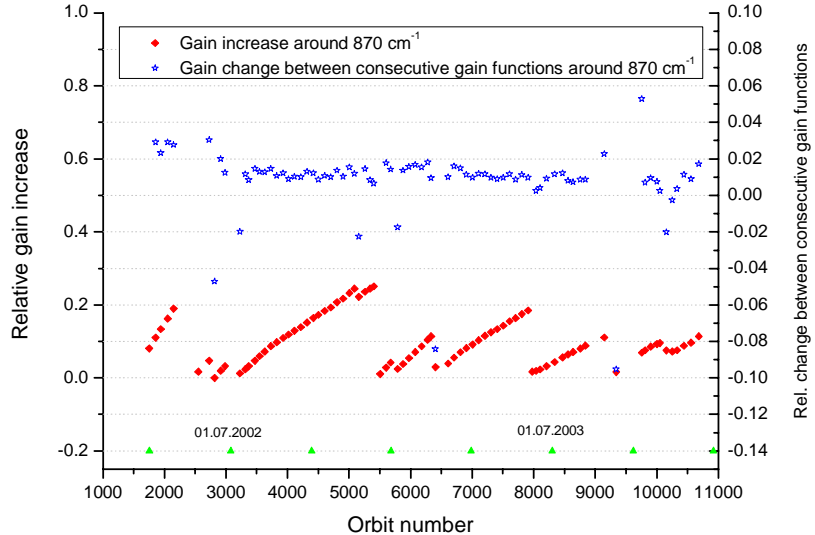

Fig. 4. Overall gain change with respect to orbit 2815 (left) and gain change between consecutive gain functions (right).

photon flux (detector curve). The non-linearity depends on the specific detector material, detector temperature, and age.

As explained in Sect. 2.4.4 non-linearity correction is carried out by scaling the measured interferograms with factors that are dependent on the incident photon flux. These factors are calculated from the non-linearity characterization of the detectors and the actual incident photon flux. The corresponding point on the detector curve is given by the sum of the background photon flux and the unmodulated target photon flux (atmospheric scene, blackbody). The background photon flux is determined by the instrument temperature and emissivity.

The non-linearity of the detectors was characterized onground in a vacuum thermal chamber by using a calibrated blackbody at different temperatures. Since the in-flight instrument temperatures are variable and differ from test conditions and furthermore the instrument transmittance may be different, too (contamination with ice - see also Sect. 4.3.1, aging of optics), an in-flight detector characterization was intended. Unfortunately, it turned out that the on-ground characterization procedure could not be used in-flight due to the very limited temperature range of the on-board calibration blackbody. Up to now the on-ground data are used for detector non-linearity correction.

A new in-flight characterization method was developed utilizing additional non-linearity information from out-ofband spectral artefacts which only are accessible in a special instrument measurements mode, the "raw data mode", where no digital filtering and decimation is applied. Non-linearity characterization can be achieved by combining this information with the in-flight blackbody measurements at different temperatures (Wagner and Birk, 2005).

The new method was applied to raw data mode measurements and blackbody measurements at different temperatures carried out in the commissioning phase. It should be noted that these are special measurements which are carried out occasionally. Figure 5 shows the differences in calibrated scene radiance levels in percent when using the on-ground and new in-flight characterization. The differences depend on the integrated photon flux shown on the $\mathrm{x}$-axis in relative units and the spectral photon flux/radiance level on the yaxis. For channel A2 perfect agreement is found while for all other channels for weak spectral signals and high integrated photon flux (low tangent altitude, cloudy scenes) differences up to $4 \%$ occur. This result shows the magnitude of the error caused by application of the on-ground detector non-linearity characterization. The actual error may differ since as mentioned earlier the characterization depends on instrument and detector temperature, ice contamination, aging of optics and detectors.

Over the lifetime of MIPAS a number of raw data mode measurements have been recorded as well as a few measurements at different blackbody temperature. Due to the very long time constant for stabilization of the blackbody temperature such measurements are very time consuming leading to the small number of data sets available. The raw data measurements in combination with an instrument model calculating the various photon fluxes on the detector allow determining the appropriate detector characterization. An accurate in-flight characterization of the detector non-linearity will reduce the systematic error on the calibrated scene and is subject of future investigations.

\subsection{Spectral calibration}

The auxiliary data file MIP_CS1_AX contains the spectral correction factor (SCF) that compensates for variations in the instrument metrology (e.g., aging of the laser). The laser is a single-mode $1.3 \mu \mathrm{m}$-diode laser, which does not have an absolute frequency stabilisation but is designed to have a good short term frequency stability (less than $50 \mathrm{MHz}$ drift over $75 \mathrm{~s})$. The initial spectral factor is applied to the first scans scene measurement data. A new spectral calibration calculation is performed every 4 elevation scans while processing a L0 product. This new spectral correction factor is applied to subsequent scene measurement data by the L1B processor. The MIP_CS1_AX is updated every week and after a long detectors/cooler switch-off or after a long unavailability period. Figure 6 gives the variation trend from May 2002 to December 2003. An overall decrease of the SCF can be observed. This is a nominal situation that is due to the aging of the laser metrology. The spectral calibration is very stable and the variations are less than $5 \mathrm{ppm}$ over the first year of operation.

\subsection{Instrument Line Shape}

The instrumental line shape (ILS) is the area-normalized spectral response function of the spectrometer. In general, the response function varies as function of the spectral position, and therefore should be denoted as $\operatorname{ILS}\left(\Delta \sigma, \sigma_{0}\right)$. When 

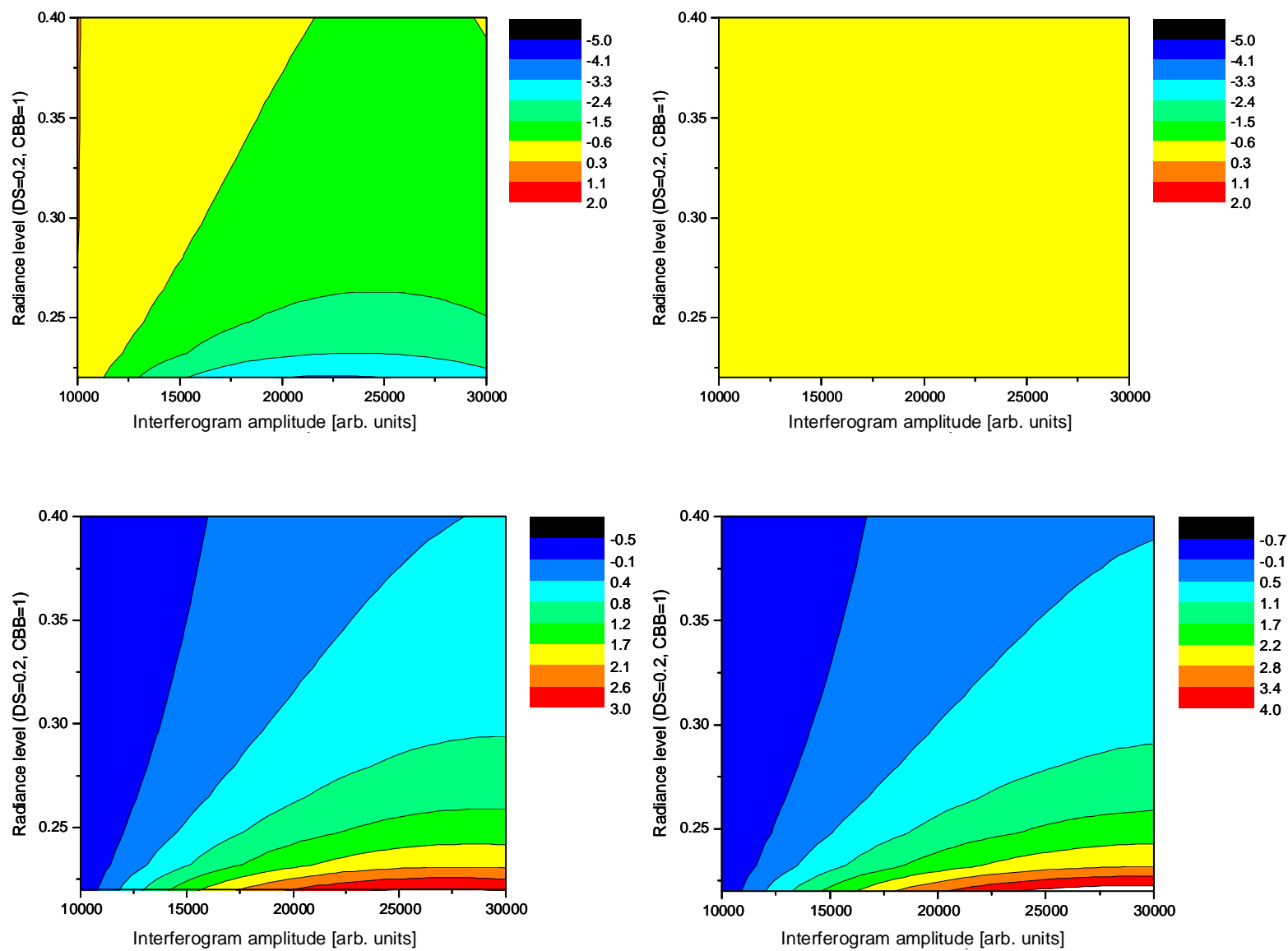

Fig. 5. Radiometric differences for the four non-linear detectors (A1, A2, B1, and B2 from left to right, top to bottom) in percent between new method and currently used on-ground characterization. $x$-axis: Estimate of integrated photon flux, y-axis: relative spectral intensity. The spectral intensity of the calibration blackbody is scaled to 1 , the spectral intensity of the deep space view is around 0.2 .

a monochromatic spectral line at position $\sigma_{0}$ enters the instrument, the ordinate value of the area-normalized response at $\sigma$ is $\operatorname{ILS}\left(\sigma-\sigma_{0}, \sigma_{0}\right)$.

Due to the normalization, the specification of an ILS is not affected by the calibration of the radiance axis. In contrast, there is an intimate connection with spectral shift: Any wave-number dependent spectral shift which occurs in the measurement can in principle be incorporated in the ILS. However, introduction of a separate shift function leads to a more transparent treatment, because spectral drifts occur on timescales of a single orbit, whereas for the profound ILS attributes (peak response, half width, and asymmetry) no significant drift on timescales of years has been observed. The spectral drift is caused by wavelength changes of the interferometer reference laser, whereas the ILS attributes as defined above are dominated by a slight permanent misalignment of the interferometer.

The ILS of MIPAS is specified by means of a set of ILS variables. Since a physical model of the interferometer is available for MIPAS, the subset of model variables which

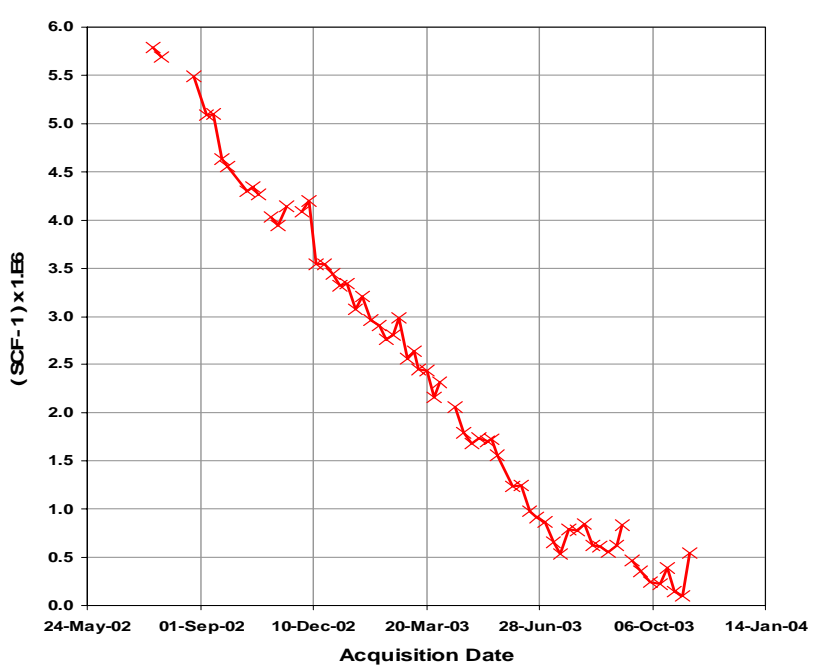

Fig. 6. Spectral Correction Factor trend from May 2002-Dec 2003. 


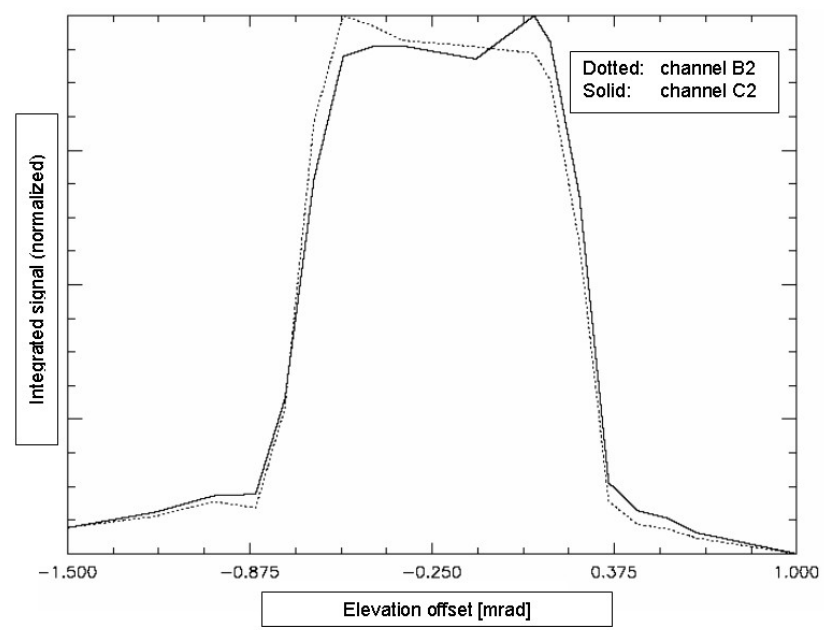

Fig. 7. Pre-launch characterization of FOV response (elevation section); detectors B2 and C2.

affect the ILS can be adjusted to approximate the ILS of the real instrument. This approach offers the advantage that the parameters are not fully ad-hoc. However, the complete set of variables cannot be retrieved unambiguously from the measured spectra, so the full set of variables has to be restricted further (to a set of fixed parameters and 2 variables). It should be noted that this reduced description is not fully physical. The suggested set of variables has been used in various studies. It is found that the loss in peak response of the unapodized ILS due to misalignment amounts $2 \%$ at $800 \mathrm{~cm}^{-1}, 5 \%$ at $1200 \mathrm{~cm}^{-1}, 8 \%$ at $1600 \mathrm{~cm}^{-1}$, and $11 \%$ at $2000 \mathrm{~cm}^{-1}$. These numbers refer to operation with full nominal resolution. The ILS degradation due to misalignment scales down when a reduced resolution is applied.

\subsection{Field of view}

Analyses of the instrument's field-of-view (FOV) response and the co-alignment of the various detector channels were performed both prior to launch and post launch. Pre-launch tests were based on a dedicated experimental setup allowing acquiring individual detector signals, with the instrument collimated input beam viewing a blackbody source. This source, equipped with a small circular aperture ("pin-hole") was moved across the field of view in azimuth and elevation direction while a set of interferograms was acquired for each discrete position. The response in the different detector channels as a function of azimuth and elevation offset was obtained by estimating the spectrally integrated signals from co-added interferograms.

Figure 7 shows a typical response pattern, obtained for detector channels B2 and C2, respectively. An in-depth analysis of the experimental data revealed that observed minor deviations of the observed response patterns from the expected shapes were due to thermal perturbations of the viewed tar-

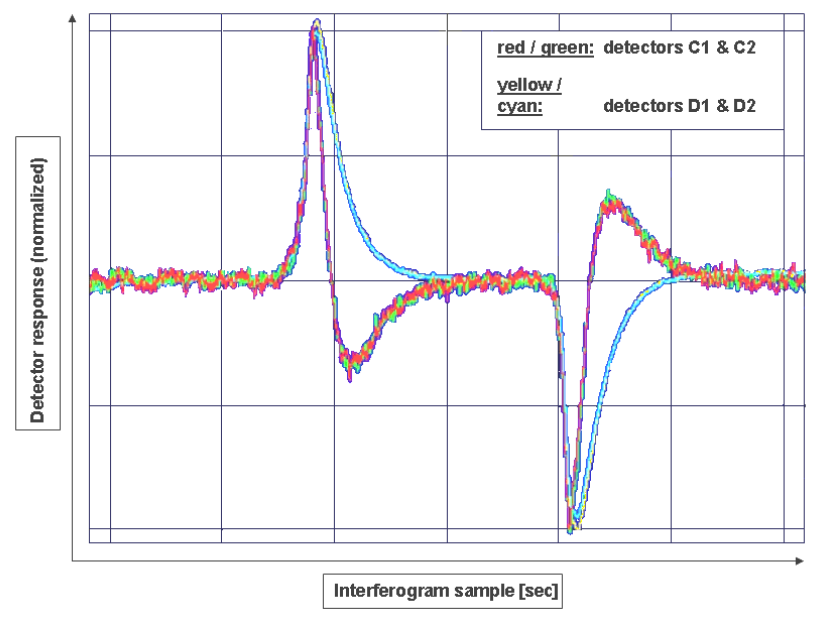

Fig. 8. In-orbit check of detector co-alignment (target: Mercury, date: 5 July 2002).

gets in the vicinity of the pin hole. This assumption was confirmed by comparing FOV patterns obtained for adjacent detector channels (A2-B1, B1-C1, etc), after reducing the signal integration to the spectral overlap for each detector pair. In conclusion, the FOV widths as well as the co-alignment between all detector channels were found within expectations.

Both co-alignment and FOV half widths of the various detector channels were verified also post-launch. For this purpose, a specific in-orbit measurement geometry was chosen in which the instrument line of sight was actively scanned across an infrared bright, "point like" source while raw mode interferograms were acquired simultaneously in all eight detector channels.

For the measurement, Mercury was chosen as a target, providing sufficiently high flux density across the wavelength

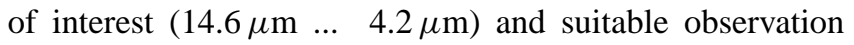
opportunities in the period of interest (June/July 2002). A schematic view of the observation geometry is provided in Fig. 1. Prior to the actual measurement the instrument LOS scan angles were adjusted such that the IR source would pass through the center of the FOV while the elevation angle is scanned at constant velocity (approx. $23.5 \mathrm{mrad} / \mathrm{s}$ ) in the positive direction, i.e. towards higher tangent heights. The overall timing was chosen such that the expected passage of the planet through the FOV occurred approx. $1 \mathrm{~s}$ after start of an appropriate interferometer sweep. The settings ensured that the actual passage occurred well before reaching the zero path difference peak and at sufficiently high tangent altitudes $(>80 \mathrm{~km})$ to avoid perturbations through atmospheric broadband emission. The result of a typical measurement is depicted in Fig. 8. The plot shows an overlay of raw interferogram signals for detectors $\mathrm{C} 1$ and $\mathrm{C} 2$, D1 and D2, covering an interval of approx. 125 samples recorded at a sampling frequency of $76.066 \mathrm{~s}^{-1}$. The resulting angular sampling 


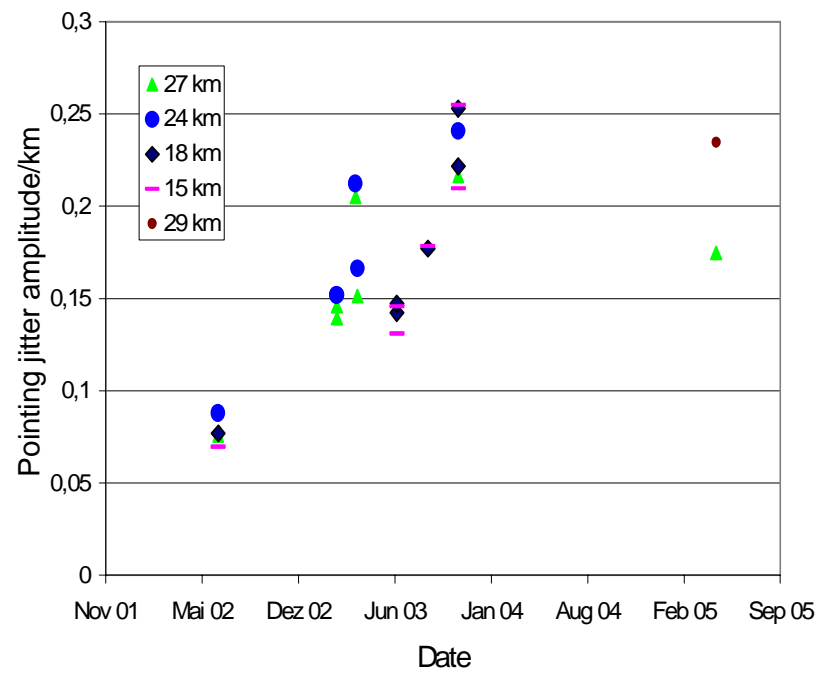

Fig. 9. Pointing jitter variations.

rate, taking into account orbital motion and scanning velocity, is $\sim 0.3 \mathrm{mrad} / \mathrm{sample}$.

The observed signal shapes reflect the response characteristics of the individual analog processing chains (AC coupling), whereas the signal rise is fully resolved for all detectors. Therefore, in order to check FOV half width and detector co-alignment, the points of inflection (POI) in the signal rise parts (left part of first positive peak and left part of second negative peak) can be analyzed. A more detailed analysis, taking into account all 8 detectors, showed that the absolute POI positions agree within $0.023 \mathrm{mrad}$ for all channels, and that the FOV half width varies between $0.788 \mathrm{mrad}$ for detectors A1 and A2 and $0.811 \mathrm{mrad}$ for detectors D1 and D2.

\subsection{Others}

\subsubsection{Forward/reverse differences}

Depending on the sweep direction (i.e. the direction of the movement of the mirrors in the interferometer), the interferograms have different phase relations and slightly different intensities. These differences should cancel out during the calibration process, which is performed for each sweep direction separately. However, some slight but systematic differences still remain in the calibrated spectra. These differences are in the order of 10 to $20 \%$ of the NESR and do not significantly influence the quality of the operational trace gas retrieval. Since one interferogram is measured for each tangent altitude, adjacent tangent altitudes are measured with opposite sweep directions. Systematic forward/reverse differences in the calibrated spectra may thus induce systematic oscillations in the retrieved trace gas profiles. An analysis of the $\mathrm{CH}_{4}$ and $\mathrm{N}_{2} \mathrm{O}$ profiles measured in November 2003 has shown that there is no evidence for a systematic forward/reverse oscillation exceeding the standard deviation of the profiles (Belotti,
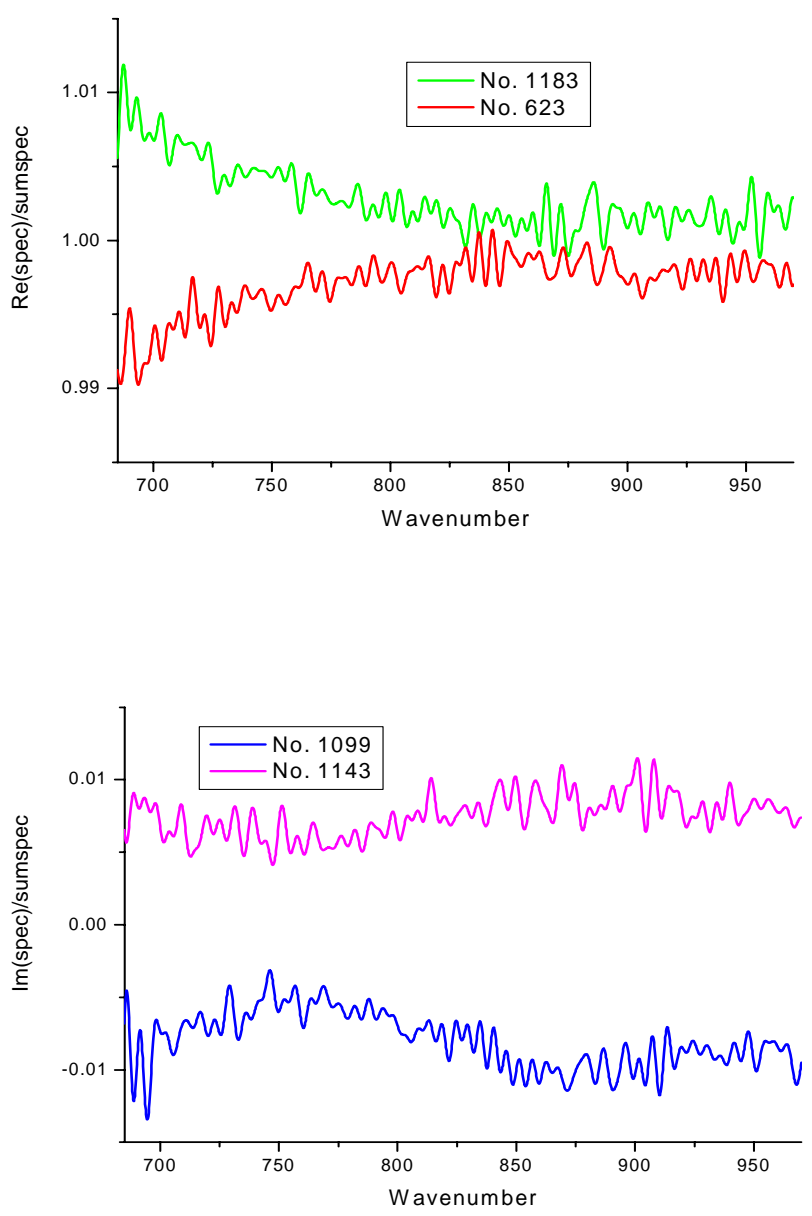

Fig. 10. Real (top) and imaginary (bottom) part of the ratio of a single calibration blackbody spectrum to the average over a sequence. Spectra were selected for maximum positive and negative deviation from 1 and 0 , respectively.

C.: Via Madonna del Piano, 10, 50019 Sesto Fiorentino (FI), Italy, personal communication, 2004)

\subsubsection{Pointing jitter}

Periodic variations of the tangent height with a frequency of $130 \mathrm{~Hz}$ have been observed in unfiltered, undecimated "raw data mode" interferograms of atmospheric scenes as sinusoidal variations of the "baseline". These variations are caused by the change of the integral radiance of a scene with tangent altitude. The pointing jitter amplitude was calculated and is shown in Fig. 9. The increase between May 2002 and later measurements may be related to the switch off of the Sterling cooler vibration cancellation, causing more vibrations. Pointing jitter leads to amplitude modulation in the interferogram and can cause ghost lines in scene spectra. From the jitter amplitude the modulation depth (modulated fraction of radiation in the interferogram) was estimated and the effect of the amplitude modulation was modelled. From this model the ghost lines should be clearly visible. However, no 
indication of ghost lines has been found in the level $1 \mathrm{~b}$ data, which is not completely understood.

\subsubsection{Microvibrations}

The radiometric calibration requires the instrument spectral gain to be constant within a calibration cycle since scene and calibration measurements are recorded at a different time. Short term gain stability was investigated from a sequence of consecutive calibration blackbody measurements by forming the ratios of single spectra to the average over the sequence. Since the spectra are complex, the ratio is also complex. In the absence of any gain variation in time its real part should be 1 and its imaginary part should be 0 . However, in the measurements, deviations of these values are observed. The magnitude of the deviations shows a periodic behaviour in time within the sequence. Figure 10 shows the maximum deviations found in the spectra. The variations can be attributed to microvibrations leading to a modulation of the sampling positions within the interferogram. The observations can be well reproduced with a model that applies modulation frequencies of about $400 \mathrm{~Hz}$ and modulation amplitudes of about $5 \mathrm{~nm}$.

As can be seen from Figure 4 the maximum variations in the gain are about $1 \%$ at maximum. Since the in-flight NESR measurement relies on rms calculation of the imaginary part of the calibrated radiance, which is expected to be 0 except for the noise, the offsets in the imaginary part lead to deteriorated NESR values. This has been remedied in the processing by subtracting the piecewise average of the imaginary part before calculating the rms. Ghost lines expected to be generated by the modulation could not be observed, possibly because the microvibrations are not necessarily monochromatic.

The microvibrations cause a pseudo-random error on the true radiance of $1 \%$ at maximum. Furthermore, an offset error is introduced, its maximum being $1 \%$ of the instrument thermal radiance which in turn is about $30 \%$ of the calibration blackbody radiance.

\section{Conclusions}

Overall, the MIPAS performance is very good and the data produced are of high quality. Very few updates were needed to the original algorithms developed for the L1B processor. NESR and radiometric accuracy requirements are met. The geolocation that depends mainly on the platform attitude control is not within requirement but very close. Instrumentation characterizations were done on-ground, verified during commissioning phase and are periodically monitored during in-flight operation. The characterizations have improved the understanding of the instrument. In most situations, a radiometric accuracy of 1 to $2 \%$ can be achieved. The most critical issue for the radiometric accuracy is the detector nonlinearity correction.

Work is still in progress to improve and refine the L1B algorithm processor in order to bring data beyond the initial requirements e.g., pointing, calibration, forward/reverse differences. Finally, high quality spectra produced by MIPAS will bring essential information to improve our knowledge of the atmospheric phenomena.

Acknowledgements. The authors wish to acknowledge the many contributors to the MIPAS L1B processing algorithms definition and validation. We especially thank the MIPAS Quality Working Group for the efforts to improve the quality of MIPAS data results.

Edited by: P. Hartogh

\section{References}

MIPAS Level 1B Algorithm technical Baseline Document: an overview, Richard L. Lachance, Ph.D, 1999.

PO-RP-BOM-GS-0003 4I: Detailed Processing Model and Parameter Data List Document (DPM) for MIPAS level 1B Processing, 2004.

PO-TN-BOM-GS-0012: Algorithm Technical Baseline Document (ATBD) for MIPAS Level 1B Processing, 2002.

PO-ID-DOR-SY-0032: Section 8, Measurement Data Definition and Format Description for MIPAS, 2000.

Wagner, G. and Birk, M.: Improvement of DLR detector nonlinearity characterization for MIPAS/Envisat. Support to MIPAS Phase E activities Technical Note, issue 1A, 29 June 2005.

PO-IS-DMS-GS-00559: Re-engineering of mission analysis software for Envisat-1. PPF-POINTING software user manual, 2005.

Fischer, H., Birk, M., Blom, C., Carli, B., Carlotti, M., v. Clarmann, T., Delbouille, L., Dudhia, A., Ehhalt, D., Endemann, M., Flaud, J. M., Gessner, R., Kleinert, A., Koopmann, R., Langen, J., Lopez-Puertas, M., Mosner, P., Nett, H., Oelhaf, H., Perron, G., Remedios, J., Ridolfi, M., Stiller, G., and Zander, R.: MIPAS: An Instrument for Atmospheric and Climate Research, Atmos. Chem. Phys. Discuss., to be submitted, 2007.

Revercomb, H. E., Buijs, H., Howell, H. B., LaPorte, D. D., Smith, W. L., and Sromovsky, L.: Radiometric calibration of IR Fourier transform spectrometers, solution to a problem with the HighResolution Interferometer Sounder, Appl. Opt., 27, 3210-3218, 1988.

Endemann, M.: MIPAS Instrument Concept and Performance, Proceedings of European symposium on atmospheric measurements from space, Noordwijk, 19-22 January 1999, WPP-161, 29-43, 1999.

Fischer, H., Blom, C., Oelhaf, H., Carli, B., Carlotti, M., Delbouille, L., Ehhalt, D., Flaud, J.-M., Isaksen, I., Lopez-Puertas, M., McElroy, C. T., and Zander, R.: Envisat - MIPAS, the Michelson Interferometer for Passive Atmospheric Sounding; An instrument for atmospheric chemistry and climate research, ESA SP1229, edited by: Readings, C. and Harris, R. A., European Space Agency, Noordwijk, The Netherlands, 2000.

Aubertin, G., Leroux, J., and Perron, G.: MIPAS L1B Processing Input/Output Data Definition, Technical ESA report, PO-TNBOM-GS-0010 4E, 2002. 